\title{
Multiphoton propagation eigenmodes for sum-frequency generation
}

\author{
Graeme Scott Docherty-Walthew, Kyle Ballantine, Michael Mazilu* \\ SUPA, School of Physics and Astronomy, \\ University of St. Andrews, St. Andrews KY16 9SS, United Kingdom
}

\begin{abstract}
In linear optics, modes correspond to a set of fields that are mutually orthogonal, however, in the nonlinear regime the principle of linear superposition is no longer valid and consequently, the method of mode decomposition breaks down. This is the case when considering sum-frequency generation in a nonlinear crystal. Here, we use the non-depleting pump approximation to describe the nonlinear interaction with low-intensity multiphoton fields. We show that the description of multiphoton fields using the propagation eigenmodes simplifies the Fock state quantum behaviour of the system while remaining equivalent to the standard approach.
\end{abstract}

Keywords: eigenmodes, quantum-optical networks, perturbation approach, single photons

\section{Introduction}

Decomposing fields into modes is a well known technique used to solve many problems in the physical sciences. In optics, the decomposition in modes has been used to describe light propagating in laser cavities [1, photonic crystals [2], optical fibres and waveguides [3]. In general, these modes correspond to a set of mutually orthogonal fields that do not interact with one another as they propagate. If, however, the fields propagating in the system are of sufficient intensity then higher-order dipole interactions can no longer be neglected 4. Due to these nonlinear dipole interactions, the principle of linear superposition breaks down and the mode decomposition used in linear optics is no longer valid. Here, we consider the interaction between fields of negligible intensity and a single high-intensity background field. This enables us to restore the principle of linear superposition for the low-intensity perturbation fields and to re-introduce the concept of orthogonal modes to these systems. Unlike the fields in linear optics, these perturbation fields interact with one another via the high-intensity background field.

In general, the propagation of light through a

\footnotetext{
${ }^{*}$ Corresponding author: Michael Mazilu

Email address: michael.mazilu@st-andrews.ac.uk (Michael Mazilu )
}

Preprint submitted to Journal of LATEX Templates linear optical system is fully described by a scattering matrix [5]. This is also the case for the perturbation fields propagating through the nonlinear crystal. In this case, the effect of the crystal can be thought of as a multiwavelength beam splitter. In the context of quantum optics, this scattering matrix also characterises the input-output relation of creation and annihilation operators of the corresponding classical fields where the field amplitudes are exchanged for probability amplitudes [6]. The quantum description of these optical systems has been studied extensively in recent years owing to their close link to the study of quantum computing, information and communication 17, 8, 9. Further, the effective Hamiltonian describing the evolution of quantum states in optical networks can be derived directly from the classical scattering matrix that characterises the system [10, 11].

In this paper, we study three-wave mixing in the context of a linear optical network in which two perturbation fields interact as they propagate. This interaction is mediated through the high intensity pump field and the nonlinear material. By quantising the perturbation fields, we introduce a unitary scattering operator that describes the evolution of Fock states as they propagate. The main result of this paper is to demonstrate that, by representing the perturbation fields using propagation eigenmodes, the Fock states associated with these

March 2, 2020 
modes are eigenstates of the unitary scattering operator describing their quantum behaviour.

\section{Field Decomposition and Scattering Ma- trix}

We start with a set of coupled equations describing three-wave mixing processes of monochromatic fields in nonlinear materials [12]:

$$
\begin{aligned}
-i \partial_{z} E_{b}(x, y, z) & =\frac{1}{2 k_{1}} \nabla^{2} E_{b}(x, y, z), \\
-i \partial_{z} F_{2}(x, y, z) & =\frac{1}{2 k_{2}} \nabla^{2} F_{2}(x, y, z) \\
& +\chi_{2} E_{b}^{*}(x, y, z) F_{3}(x, y, z) e^{-i \Delta k z}, \\
-i \partial_{z} F_{3}(x, y, z) & =\frac{1}{2 k_{3}} \nabla^{2} F_{3}(x, y, z) \\
& +\chi_{3} E_{b}(x, y, z) F_{2}(x, y, z) e^{i \Delta k z},
\end{aligned}
$$

where $\Delta k=k_{3}-k_{2}-k_{b}$ is the material wave vector mismatch and $F_{2}(x, y, z)$ and $F_{3}(x, y, z)$ are monochromatic electric fields oscillating at $\omega_{2}$ and $\omega_{3}$ respectively. The field $E_{b}(x, y, z)$ is a highintensity pump oscillating with frequency $\omega_{1}=$ $\omega_{3}-\omega_{2}$ which evolves independent of the fields $F_{\tau}$ for $\tau=2,3$. Indeed, we assume that the pump field is of sufficient high total intensity such that the interaction term $F_{2}(x, y, z)^{*} F_{3}(x, y, z)$ can be considered negligible, rendering the pump non-depleted:

$$
\begin{aligned}
& \iint \mathrm{d} x \mathrm{~d} y\left|E_{b}(x, y, 0)\right| \gg \\
& \iint \mathrm{d} x \mathrm{~d} y\left|\chi_{1} F_{2}^{*}(x, y, 0) F_{3}(x, y, 0)\right| \Delta z,
\end{aligned}
$$

where $\Delta z$ is the crystal thickness.

The interaction strength is given by $\chi_{\tau}=\frac{\chi^{(2)} \omega_{\tau}}{n_{\tau} c}$ where $\chi^{(2)}$ is the second order susceptibility of the system. We can expand the fields as

$$
F_{\tau}=\sum_{j} \sqrt{\frac{\omega_{\tau}}{n_{\tau}}} a_{\tau, j}(z) f_{\tau, j}(x, y) .
$$

where the function, $\sqrt{\omega_{\tau} / n_{\tau}} f_{\tau, j}(x, y)$ form a complete, orthonormal basis with

$$
\begin{array}{r}
\iiint \mathrm{d} x \mathrm{~d} y \mathrm{~d} t e^{-i\left(\omega_{\sigma}-\omega_{\tau}\right) t} \sqrt{\frac{\omega_{\tau}}{n_{\tau}}} \sqrt{\frac{\omega_{\sigma}}{n_{\sigma}}} \\
f_{\tau, i}^{*}(x, y) f_{\sigma, j}(x, y)=\delta_{\sigma \tau} \delta_{i j} .
\end{array}
$$

If we input the expansion, Eq. (5), into Eqs. (2) and (3) we derive our equations of evolution in $\mathrm{co}^{-}$ efficient form:

$$
\begin{aligned}
& -i \partial_{z} a_{2, j}(z)=\sum_{k} \omega_{2, j k} a_{2, k}(z)+g_{j k}(z) a_{3, k}(z), \\
& -i \partial_{z} a_{3, j}(z)=\sum_{k} \omega_{3, j k} a_{3, k}(z)+g_{k j}^{*}(z) a_{2, j}(z),
\end{aligned}
$$

with the matrices:

$$
\omega_{\tau, j k}=\iint \frac{1}{2 k_{\tau}} f_{\tau, k}^{*}(x, y) \nabla^{2} f_{\tau, j}(x, y) \mathrm{d} x \mathrm{~d} y,
$$

$g_{j k}(z)=\chi \iint E_{b}^{*}(x, y, z) f_{2, j}^{*}(x, y) f_{3, k}(x, y) e^{-i \Delta k z} \mathrm{~d} x \mathrm{~d} y$

where $E_{b}(x, y, z)$ is a solution of Eq. (1) and $\chi=$ $\frac{\chi^{(2)}}{c} \sqrt{\left(\omega_{2} \omega_{3}\right) /\left(n_{2} n_{3}\right)}$. The matrix $\omega_{\tau, j k}$ is a Hermitian phase term with $\omega_{\tau, j k}=\omega_{\tau, k j}^{*}$ and $g_{j k}(z)$ describes the interaction of the modes mediated by the non-depleting background field, $E_{b}(x, y, z)$.

The evolution of the coefficients given by Eqs. (7) are written in terms of a scattering matrix as

$$
\left(\begin{array}{c}
a_{2, k}^{\prime} \\
a_{3, k}^{\prime}
\end{array}\right)=\mathbf{S}\left(\begin{array}{c}
a_{2, k} \\
a_{3, k}
\end{array}\right)
$$

where $a_{\tau, k}$ are the field amplitudes of the modes at the input plane and $a_{\tau, k}^{\prime}$ are the amplitudes of the corresponding output modes and $\mathbf{S}=$ $\exp \left(\int_{z} i \mathbf{P}(z) \mathrm{d} z\right)$ with

$$
\mathbf{P}(z)=\left(\begin{array}{cc}
\omega_{2, j k} & g_{j k}(z) \\
g_{k j}^{*}(z) & \omega_{3, j k}
\end{array}\right) .
$$

In general the matrix $g_{j k}$ is not Hermitian, however, the matrix $\mathbf{P}(z)$ is Hermitian, i.e $\mathbf{P}(z)=$ $\mathbf{P}(z)^{\dagger}$, where $\mathbf{P}(z)^{\dagger}$ is the Hermitian conjugate. Consequently, the scattering matrix is unitary

$$
\begin{aligned}
& \mathbf{S}^{\dagger} \mathbf{S}=\exp \left(\int_{z} i \mathbf{P}(z) \mathrm{d} z\right) \exp \left(\int_{z}-i \mathbf{P}(z)^{\dagger} \mathrm{d} z\right) \\
& =\exp \left(\int_{z} i \mathbf{P}(z) \mathrm{d} z\right) \exp \left(\int_{z}-i \mathbf{P}(z) \mathrm{d} z\right)=\mathbf{I},
\end{aligned}
$$

where $\mathbf{I}$ is the identity matrix of the same dimensions as $\mathbf{S}$. From a physical perspective the unitarity of the scattering matrix is an expression of the 
conservation of the quantity

$$
\begin{aligned}
& \Phi=\sum_{\tau} \frac{\mathscr{I}_{\tau}}{\hbar \omega_{\tau}}=\sum_{\tau} \frac{n_{\tau} c \epsilon_{0}}{2 \hbar \omega_{\tau}} F_{\tau}^{*}(x, y, z) F_{\tau}(x, y, z) \\
& =\sum_{\tau, j} \frac{c \epsilon_{0}}{2 \hbar} a_{\tau, j}(z)^{*} a_{\tau, j}(z) f_{\tau, j}^{*}(x, y, z) f_{\tau, j}(x, y, z),
\end{aligned}
$$

where $\mathscr{I}_{\tau}=\frac{1}{2} n_{\tau} \epsilon_{0} c F_{\tau}^{*}(x, y, z) F_{\tau}(x, y, z)$ is the total intensity of the field $F_{\tau}$ and $\Phi$ is the photon flux 13 .

\section{Effective Hamiltonian and Scattering Op- erator}

Considering the field expansion described in Eq. (5), we can quantise the system by promoting the coefficients to operators:

$$
\begin{aligned}
& a_{\tau, j} \rightarrow \hat{a}_{\tau, j}, \\
& a_{\tau, j}^{*} \rightarrow \hat{a}_{\tau, j}^{\dagger} .
\end{aligned}
$$

The evolution of these operators is the same as the field amplitudes as described in Eqs. (7) with the canonical commutation relations

$$
\left[\hat{a}_{\tau, i}, \hat{a}_{\sigma, j}^{\dagger}\right]=\delta_{i j} \delta_{\tau \sigma} .
$$

To simplify the notation in the commutator, we drop the first index $\tau$ that distinguishes between the two wavelengths giving, $\left(\hat{a}_{1}, \ldots, \hat{a}_{\tau}, \ldots, \hat{a}_{m}\right)=$ $\left(\hat{a}_{2,1}, \ldots, \hat{a}_{2, m_{2}} ; \hat{a}_{3,1}, \ldots, \hat{a}_{3, m_{3}}\right)$, where $m_{2}+m_{3}=$ $m$. The numbers $m_{3}$ and $m_{2}$ are the number of modes in the decomposition, Eq. (5), for each field. The commutation relations in this simplified notation are, $\left[\hat{a}_{i}, \hat{a}_{j}^{\dagger}\right]=\delta_{i j}$.

In this case, we have the scattering matrix Eq. 10 linking the annihilation operators of the output modes $\hat{\mathbf{A}}^{\prime}$ to the annihilation operators of the input modes $\hat{\mathbf{A}}$ as

$$
\hat{\mathbf{A}}^{\prime}=\mathbf{S} \hat{\mathbf{A}},
$$

with $\hat{\mathbf{A}}$ and $\hat{\mathbf{A}}^{\prime}$ defined as the column vectors, $\hat{\mathbf{A}}=\left(\hat{a}_{1}, \ldots, \hat{a}_{m}\right)^{\top}$ and $\hat{\mathbf{A}}^{\prime}=\left(\hat{a}_{1}^{\prime}, \ldots, \hat{a}_{m}^{\prime}\right)^{\top}$, where the symbol $\top$ denotes the transpose. Similarly, the input output creation operators are linked via the Hermitian conjugated Eq. (16).

In the Schrödinger picture of quantum mechanics input states $\left|\psi_{\text {in }}\right\rangle$ evolve into an output states, $|\psi\rangle_{\text {out }}$, by acting on the input states with a unitary operator, $\hat{S}$, as

$$
\left|\psi_{\text {out }}\right\rangle=\hat{S}\left|\psi_{\text {in }}\right\rangle \text {. }
$$

According to the Heisenberg picture, the state vectors are fixed and the operators evolve as, $\hat{\mathbf{A}}^{\prime}=$ $\hat{S}^{\dagger} \hat{\mathbf{A}} \hat{S}$, which is equivalent to the Schrödinger picture [14]. Using this together with Eq. (16), we have

$$
\mathbf{S} \hat{\mathbf{A}}=\hat{S}^{\dagger} \hat{\mathbf{A}} \hat{S} .
$$

As the matrix, $\mathbf{S}$, is unitary we can introduce the Hermitian matrix, $\mathbf{H}=-i \ln \mathbf{S}$, and define the effective Hamiltonian with the use of a JordanSchwinger map 15

$$
\hat{H}_{\mathrm{eff}}=\left(\hat{a}_{1}^{\dagger}, \ldots, \hat{a}_{m}^{\dagger}\right) \mathbf{H}\left(\begin{array}{c}
\hat{a}_{1} \\
\vdots \\
\hat{a}_{m}
\end{array}\right)=\hat{\mathbf{A}}^{\dagger} \mathbf{H} \hat{\mathbf{A}}
$$

where the Hermiticity of $\hat{H}_{\text {eff }}$ follows from the Hermiticity of $\mathbf{H}$. This matrix is related to the classical scattering matrix via the exponential map. Similarly, the effective Hamiltonian is related to the unitary scattering operator via the unitary-Hermitian homomorphism [11], $\hat{S}=\exp \left(i \hat{H}_{\text {eff }}\right)$, satisfying the Schrödinger equation, Eq. (17), and, the condition given by Eq. (18). This approach is equivalent to the one presented for photon conserving unitary scattering matrices [1] and can be expanded to more general optical networks [10, 16.

The Fock state basis elements are $|\psi\rangle=$ $\left|n_{1}, \ldots, n_{m}\right\rangle$ where $n_{j}$ is the total number of photons in the $j^{t h}$ mode. The operators $\hat{a}_{j}$ and $\hat{a}_{j}^{\dagger}$ acting on these basis states are creation and annihilation operators with the standard raising and lowering relations:

$$
\begin{array}{r}
\hat{a}_{j}\left|n_{1}, \ldots, n_{j}, \ldots, n_{m}\right\rangle \\
=\sqrt{n_{j}}\left|n_{1}, \ldots, n_{j}-1, \ldots, n_{m}\right\rangle, \\
\hat{a}_{j}^{\dagger}\left|n_{1}, \ldots, n_{j}, \ldots, n_{m}\right\rangle \\
=\sqrt{n_{j}+1}\left|n_{1}, \ldots, n_{j}+1, \ldots, n_{m}\right\rangle .
\end{array}
$$

Using the above properties the states, $\left|n_{1}, \ldots, n_{m}\right\rangle$, written in terms of the creation operators are

$$
\left|n_{1}, \ldots, n_{m}\right\rangle=\prod_{k=1}^{m}\left(\frac{\hat{a}_{k}^{\dagger n_{k}}}{\sqrt{n_{k} !}}\right)|0, \ldots, 0\rangle
$$


which evolve to the output plane as

$\hat{S}\left|n_{1}, \ldots, n_{m}\right\rangle=\prod_{k=1}^{m} \frac{1}{\sqrt{n_{k} !}}\left(\sum_{j=1}^{m} \mathbf{S}_{k j}^{*} \hat{a}_{j}^{\dagger}\right)^{n_{k}}|0, \ldots, 0\rangle$,

showing a link between the evolution of the photon states, $\left|n_{1}, \ldots, n_{m}\right\rangle$, and the evolution of the fields used to describe the photons. This is most apparent when the system consists of a single photon such that Eq. 23 is now written

$$
\hat{S}\left|0, \ldots, n_{k}=1, \ldots, 0\right\rangle=\left(\sum_{j=1}^{m} \mathbf{S}_{k j}^{*} \hat{a}_{j}^{\dagger}\right)|0, \ldots, 0\rangle,
$$

where the input states on the left hand side will have zero photons on all of the modes except the $k^{t h}$ mode. We thus observe that in the case of a single photon in the system the scattering operator, $\hat{S}$ reduces to the classical scattering matrix applied to the creation operators.

\section{Propagation Eigenmodes}

As the description of the Fock states directly depends on the scattering matrix, $\mathbf{S}$, the choice of basis used to represent the classical fields will determine the decomposition of the Fock states in this basis.

As shown in Eq. 12, the scattering matrix is unitary and therefore can be represented using an eigendecomposition of the form

$$
\mathbf{S}=\mathbf{U D U}^{\dagger}
$$

where $\mathbf{U}$ corresponds to an eigenvector matrix with, $\mathbf{U}^{\dagger} \mathbf{U}=\mathbf{U U}^{\dagger}=\mathbf{I}$ and $\mathbf{I}$ is the identity matrix. In general the eigenvectors in $\mathbf{U}$ correspond to orthogonal modes that have coupled components which oscillate at $\omega_{2}$ and at $\omega_{3}[17$.

Using the eigendecomposition of the scattering matrix we define the eigenmode annihilation and creation operators, $\hat{\mathbf{B}}$ and $\hat{\mathbf{B}}^{\dagger}$, at the input and output as:

$$
\hat{\mathbf{B}}^{\prime}=\mathbf{U}^{\dagger} \hat{\mathbf{A}}^{\prime}, \quad \hat{\mathbf{B}}=\mathbf{U}^{\dagger} \hat{\mathbf{A}},
$$

with $\hat{\mathbf{B}}^{\dagger}$ defined by the Hermitian conjugates of Eq. (26).

As with the vector, $\hat{\mathbf{A}}$, we define $\hat{\mathbf{B}}=$ $\left(\hat{b}_{1}, \ldots, \hat{b}_{m}\right)^{\top}$, as the annihilation operator for the eigenmodes at the input with elements

$$
\hat{b}_{k}=\sum_{j=1}^{m} \mathbf{U}_{j k}^{*} \hat{a}_{j}
$$

Similarly, we define $\hat{\mathbf{B}}^{\prime}=\left(\hat{b}_{1}^{\prime}, \ldots, \hat{b}_{m}^{\prime}\right)^{\top}$ as the operator associated with the output eigenmodes. Using this, we can introduce a new scattering matrix for the eigenmode operators starting with Eq. 10 which we rewrite as

$$
\hat{\mathbf{A}}^{\prime}=\left(\mathbf{U D U}^{\dagger}\right) \hat{\mathbf{A}},
$$

which can then be rearrange as

$$
\mathbf{U}^{\dagger} \hat{\mathbf{A}}^{\prime}=\left(\mathbf{U}^{\dagger} \mathbf{U} \mathbf{D} \mathbf{U}^{\dagger}\right) \hat{\mathbf{A}} \text {. }
$$

and using the transformation defined in Eq. 26 our input-output relationship is simplified as

$$
\hat{\mathbf{B}}^{\prime}=\mathbf{D} \hat{\mathbf{B}}
$$

A similar expression can be written for the creation operators with the Hermitian conjugate of the transformations in Eq. (26).

The eigenmodes operators introduced in Eq. (27) have bosonic commutation relations $\left[\hat{b}_{i}, \hat{b}_{j}^{\dagger}\right]=\delta_{i j}$ and are equivalent to a Bogoliubov transformation 18. of the initial creation and annihilation operators in the context of a propagation eigenmode decomposition. These operators decouple the state evolution as described in Eq. (30) as $\mathbf{D}$ is a diagonal matrix with elements $\left|D_{j j}\right|=1$. Therefore, by making a transformation of the classical field representation, the Fock states $\left|n_{1}, \ldots, n_{m}\right\rangle$ are eigenstates of the system unlike the states given by Eq. (23), which are distributed across the Fock state basis.

In short, using the eigenmode decomposition we replace the Fock state coupling by classical field coupling. Indeed, if we make use of the transformations described in Eq. (26) the evolution of the Fock states in the eigenmode basis is

$$
\begin{aligned}
\hat{S}_{b}\left|n_{1}, \ldots, n_{m}\right\rangle_{b}^{i n} & =\prod_{k=1}^{m} \frac{1}{\sqrt{n_{k} !}}\left(\sum_{j=1}^{m} D_{j k} \hat{b}_{k}^{\dagger}\right)^{n_{k}}|0, \ldots, 0\rangle \\
& =\prod_{k=1}^{m} D_{k k}^{n_{k}} \frac{1}{\sqrt{n_{k} !}} \hat{b}_{k}^{\dagger n_{k}}|0, \ldots, 0\rangle,
\end{aligned}
$$

where the subscript |\rangle$_{b}$ is introduced for states in the eigenmode basis with vacuum mode considered 
to be representation independent and where $\hat{S}_{b}$ is the scattering operator derived from the diagonal scattering matrix, D. This scattering operator, $\hat{S}_{b}$ is diagonal which we can show explicitly by substituting the input states

$$
\left|n_{1}, \ldots, n_{m}\right\rangle_{b}^{\text {in }}=\prod_{k=1}^{m} \frac{1}{\sqrt{n_{k} !}} \hat{b}_{k}^{\dagger n_{k}}|0, \ldots, 0\rangle,
$$

into Eq. (31) such that the matrix elements of the scattering operator are

$$
\left\langle n_{1}^{\prime}, \ldots,\left.n_{m}^{\prime}\right|_{b} ^{i n} \hat{S}_{b} \mid n_{1}, \ldots, n_{m}\right\rangle_{b}^{i n}=\prod_{k=1}^{m} D_{k k}^{n_{k}} \delta_{n^{\prime} n} .
$$

Here we have used the orthogonality of the Fock states in which the function $\delta_{n^{\prime} n}=1$ if $n_{j}^{\prime}=n_{j} \forall j$ and 0 otherwise.

Given the link between the operators, $\hat{a}$ and $\hat{b}$, we can write the states with respect to either of the operators in Fock space

$$
\begin{aligned}
\left|n_{1}, \ldots, n_{m}\right\rangle_{b}^{\text {in }} & =\prod_{k=1}^{m} \frac{1}{\sqrt{n_{k} !}} \hat{b}_{k}^{\dagger n_{k}}|0, \ldots, 0\rangle \\
& =\prod_{k=1}^{m} \frac{1}{\sqrt{n_{k} !}}\left(\sum_{j=1}^{m} U_{k j} \hat{a}_{j}^{\dagger}\right)^{n_{k}}|0, \ldots, 0\rangle .
\end{aligned}
$$

This expression gives us a direct way to transform between both representations in the context of Fock states and ensures the photon states correspond to the same physical states regardless of how they are represented.

\section{Numerical Example}

As a numerical application, we model the interaction between three fields $\left(\lambda_{b}=1064 \mathrm{~nm}, \lambda_{2}=740\right.$ and $\lambda_{3}=436.5 \mathrm{~nm}$ ) propagating through a nonlinear bulk crystal with a length of $1 \mathrm{~mm}$. In particular, we consider a Potassium Dihydrogen Phosphate (KDP) crystal with a nonlinear susceptibility of $\chi_{\mathrm{eff}}^{(2)}=0.568 \mathrm{pm} / \mathrm{V}$ which was found using the "SNLO" nonlinear modelling software [19] and references therein. To simplify the model, we further consider the case of type I perfect phase matching, $\Delta k=0$, in which the fields at $\lambda_{1}$ and $\lambda_{b}$ are polarised along the ordinary axis of the crystal and the field at $\lambda_{3}$ along the extraordinary axis.
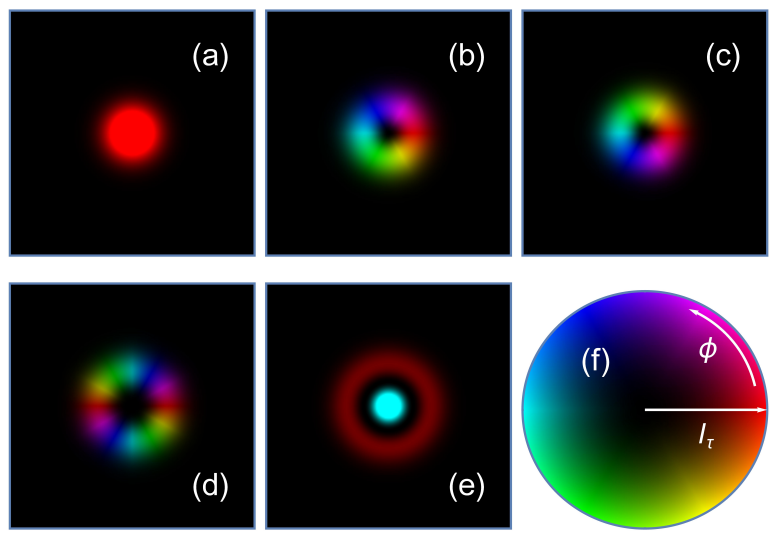

Figure 1: Modes, $f_{\tau, j}(x, y)$, for a circular waveguide with OAM (a) $\ell=0$ and $p=0$, (b) $\ell=+1$ and $p=0$, (c) $\ell=-1$ and $p=0$, (d) $\ell=2$ and $p=0$ and (e) $\ell=0$ with $p=1$, represented by the false colour map (f) with Hue showing intensity, $I_{\tau}$, and colour showing phase, $\phi$.

Here, we choose a basis set comprised of Laguerre-Gaussian (LG) modes, a few of which are shown in figure 1 with their mathematical form shown in Appendix A. This basis set is interesting as LG beams carry orbital angular momentum $(\mathrm{OAM})$ in their helical wavefront where the amount of "twist" of the wavefront corresponds the OAM, $\ell$, of the beam [20]. The radial index, $p$, corresponds to the order of the Laguerre polynomial defining the transverse structure of the mode. The background field considered, $E_{b}(x, y, z)$, has a beam waist $w_{b}=20 \lambda_{b}$, radial index $p=0$ and an average power of $10 \mathrm{nW}$ which equivalent to $10^{10}$ photons $\mathrm{s}^{-1}$. Therefore, the background field will not be perturbed by the presence of the other two fields while it would be possible to detect single photons when using suitable notch filters.

To visualise the results from our simulation, we truncate the Hilbert space defined by the LaguerreGaussian modes to a maximal index of $2 p_{\tau}+\ell_{\tau}=2$ for $\tau=2,3$ and consider a background field with $\ell_{b}=0$. This gives a total of 6 spatial degrees of freedom per perturbation field. The classical scattering matrix in this basis is illustrated in figure 2 and has a block diagonal structure. This is due to the selection rules associated with the OAM of the fields, $\ell_{3}-\ell_{2}=\ell_{b}$, which for a background field with $\ell_{b}=0$ reduces to, $\ell_{2}=\ell_{3}$.

This scattering matrix defines a scattering operator as outlined in section 3 which is illustrated in figure $3(\mathrm{a})$. The scattering operator is calculated up to a maximum total photon number of $N_{\max }=2$ 


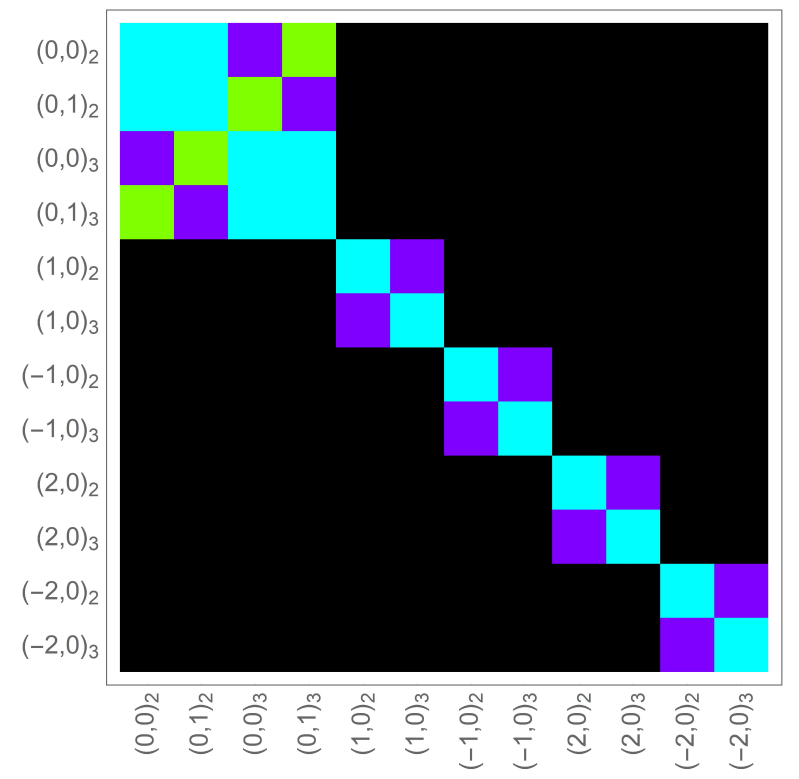

Figure 2: Classical scattering matrix, $\mathbf{S}$, for a basis of 12 fields with indices $(\ell, p)_{\tau}$ represented on a false colour space shown in figure 1 (f).

where the matrix blocks highlighted with the grid lines correspond to the $n$ photon Hilbert spaces. As shown in Eq. 13 the number of photons is conserved and therefore there are no amplification or loss mechanisms in the system. As a consequence the $n$ photon Hilbert spaces do not interact with one another. Therefore, we observe the scattering operator to be block diagonal in the total photon number representation. Each of these total photon number blocks are further block diagonal due to the symmetries associated with the OAM as illustrated in figure 2 .

The eigenmodes of the scattering matrix, figure 2 , correspond to a set of orthogonal fields that do not interaction with one another as they propagate. The scattering matrix that transforms these eigenmodes from the input to the output, $\mathbf{D}$, is therefore diagonal. Using this new scattering matrix we can define the quantum behaviour of the system with the unitary operator, $\hat{S}_{b}$, illustrated in figure 3(b). As shown in Eq. 33, if the scattering matrix transforming the classical modes is diagonal then the corresponding scattering operator will also be diagonal. Therefore, by utilising the eigenmode transformation defined in, Eq. (26), the Fock states associated with the eigenmodes of $\mathbf{S}$ will evolves as eigenstates of the system. Consequently, in this eigenmode basis, a single photon coupled to one of the eigenmodes will remain coupled to that eigenmode as it evolves through the nonlinear material. Further details about the numerical implementation of the example presented here along with a secondary example with $\ell_{b}=+1$ can be found in Appendix B and Appendix C

\section{Discussion}

Using a high intensity, non-depleting background field we linearise the equations describing threewave mixing in nonlinear materials. In this linearised system the transformation of input mode amplitudes to the output amplitudes is fully characterised by a scattering matrix. In the context of quantum optics this scattering matrix also describes the input-output relation of the creation and annihilation operators. Using the unitaryHermitian homomorphism, we can define a unitary operator that describes the quantum behaviour of the system that is dependent on the initial scattering matrix considered.

Using the perturbation approach, we have restored the principle of linear superposition to the nonlinear system. This enabled us to define of a set of orthogonal eigenmodes. These eigenmodes differ from the general modes used in linear optics as they describe a set of orthogonal fields that are distributed across multiple wavelengths. Using these eigenmodes as a representation of our fields defining the scattering matrix, we show that the evolution of the quantum photon states is decoupled. In particular, in a Fock state basis, the photon number states associated with these eigenmodes correspond to decoupled eigenstates of the quantum system.

\section{Acknowledgements}

The authors acknowledge funding from the UK Engineering and Physical Sciences Research Council (EPSRC) Grant EP/M000869/1.

\section{References}

[1] H. Kogelnik, T. Li, Laser beam and resonators, Applied Optics 5 (10) (1966) 1550-1567.

[2] P. Bienstman, R. Baets, Optical modeling of photonic crystals and VCSELs using eigenmode expansion and perfectly matched layers, Optical and Quantum Electronics 33 (2001) 327-341. 

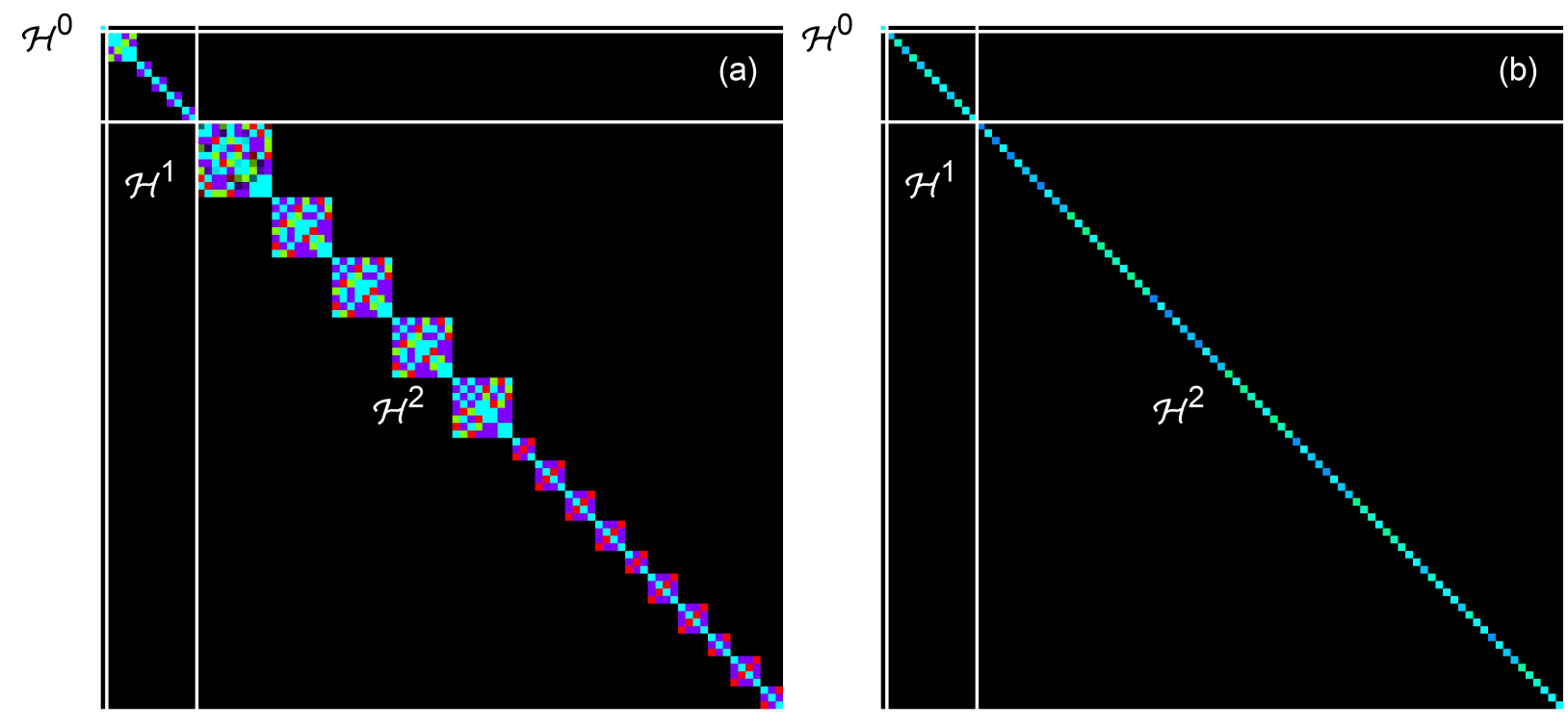

Figure 3: Unitary scattering operator (a) $\hat{S}$ and (b) $\hat{S}_{b}$ represented with false colour space with false colour map shown in in figure 1 (f). The labels $\mathcal{H}^{n}$ correspond to the the $\mathrm{n}$ photon number Hilbert space.

[3] A. S. Sudbo, Film mode matching: a versatile numerical method for vector mode field calculations in dielectric waveguides, Pure Applied Optics 2 (1993) 211-233.

[4] R. Boyd, Nonlinear Optics, 3rd Edition, Elsevier Inc., 2008.

[5] A. Wheeler, J, On the mathematical description of light nuclei by the method of resonating group structure, Physical Review 52 (11) (1937) 1107-1122.

[6] J. Skaar, J. C. G. Escartin, H. Landro, Quantum mechanical description of linear optics, American Journal of Physics 72 (2004) 1385-1391.

[7] S. Aaronson, A. Arkhipov, The computational complexity of linear optics, Proceedings of the 43rd Annual ACM Symposium on Theory of Computing 11 (2011) 333342 .

[8] S.-H. Tana, P. P. Rohded, The resurgence of the linear optics quantum interferometer recent advances \& applications, Reviews in Physics 4.

[9] M. Suda, C. Pacher, M. Peev, M. Duek, F. Hipp, Quantum interference of photons in simple networks, Quantum Inf Process 12 (2013) 1915-1945.

[10] U. Leonhardt, V. Neumaier, Explicit effective hamiltonians for general linear quantum-optical networks, Journal of Optics B: Quantum and Semiclassical Optics 6 (2004) L1-L4.

[11] C. Garcia-Escartin, J, V. Gimeno, J. Moyano-Fernndez, Multiple photon effective hamiltonians in linear quantum optical networks, Optics Communications 430 (2018) 434-439.

[12] Y. R. Shen, The Principles of Nonlinear Optics, John Wiley \& Sons, Hoboken, New Jersey, 2003.

[13] G. New, Introduction to Nonlinear Optics, Cambridge University Press, 2011.

[14] C. Madrid Casado, M, A brief history of the mathemat- ical equivalence between the two quantum mechanics, Lat. Am. J. Phys. Educ. 2 (2) (2008) 104-108.

[15] P. Aniello, C. Lupo, Exploring representation theory of unitary groups via linear optical passive devices, Open Sys. \& Information Dyn. 13 (2006) 415426.

[16] L. Braunstein, S, Squeezing as an irreducible resource, Physical Review A. 71.

[17] G. Docherty-Walthew, M. Mazilu, Nonlinear optical eigenmodes; perturbative approach, Proc. SPIE 10935, Complex Light and Optical Forces XIII 109351K.

[18] N. Bogoljubov, N, On a new method in the theory of superconductivity, Il Nuovo Cimento 7 (1958) 794-805.

[19] SNLO nonlinear optics code available from A. V. Smith, Albuquerque, NM, SNLO v71, AS-Photonics.

[20] L. Allen, M. Barnett S, J. Padgett, M, Optical Angular Momentum, CRC Press LLC, 2016.

[21] A. E. Siegman, Lasers, University Science Books, 1986.

[22] I. Wolfram Research, Mathematica, version 12.0 Edition, Wolfram Research Inc., 2019. 


\section{Appendix A. Laguerre-Gaussian modes}

Systems the exhibit circular symmetry are best solved with field profiles that are themselves circularly symmetric. A prominent example of such fields are the Laguerre-Gaussian modes [21] defined as

$$
\begin{array}{r}
E_{\ell, p}(r, \theta, z)=\frac{A_{0}}{w(z)}\left(\frac{\sqrt{2} r}{w(z)}\right)^{\ell} L_{p}^{\ell}\left(\frac{2 r^{2}}{w(z)^{2}}\right) \\
\quad \times e^{-\frac{r^{2}}{w(z)^{2}}} e^{\frac{i k r^{2}}{2 R(z)^{2}}} e^{-i \phi(z)} e^{i \ell \theta}
\end{array}
$$

where $L_{p}^{\ell}$ are the generalised Laguerre polynomials which are defined as

$$
L_{p}^{\ell}(\tau)=\frac{\tau^{-\ell} e^{\tau}}{p !} \frac{d^{p}}{d \tau^{p}}\left(e^{-\tau} \tau^{\ell+p}\right)
$$

with $\tau=x, y$. The index $\ell$ corresponds to the number of helical wavefronts of the mode and the index $p$ is the radial index corresponding to the order of the Laguerre polynomial.

\section{Appendix B. Numerical Implementation}

In this section we outline the numerical considerations not included in the main text. In section 5 we outlined an example of a truncated basis of OAM fields in a circular waveguide. In this example the Fock state space was also truncated with respect to some maximum total photon number, $N_{\max }$. As a consequence, we also truncate the creation and annihilation operators such that they are both closed operations in our finite Fock space. If we have an input state, $\left|n_{1}, n_{2}\right\rangle$, then the $\hat{a}_{2}$ and $\hat{a}_{3}$ operators should satisfy the following properties:

$$
\begin{aligned}
& \hat{a}_{2}\left|n_{1}, n_{2}\right\rangle=\sqrt{n_{1}}\left|n_{1}-1, n_{2}\right\rangle, \\
& \hat{a}_{3}\left|n_{1}, n_{2}\right\rangle=\sqrt{n_{2}}\left|n_{1}, n_{2}-1\right\rangle .
\end{aligned}
$$

If we write the output state as, $\left|n_{1}^{\prime}, n_{2}^{\prime}\right\rangle$, then using the above properties we can write the matrix elements of the annihilation operators as

$$
\begin{aligned}
\left\langle n_{1}^{\prime}, n_{2}^{\prime}\left|\hat{a}_{2}\right| n_{1}, n_{2}\right\rangle & =\sqrt{n_{1}} \delta_{n_{1}, n_{1}^{\prime}-1} \delta_{n_{2}, n_{2}^{\prime}}, \\
\left\langle n_{1}^{\prime}, n_{2}^{\prime}\left|\hat{a}_{3}\right| n_{1}, n_{2}\right\rangle & =\sqrt{n_{2}} \delta_{n_{2}, n_{2}^{\prime}-1} \delta_{n_{1}, n_{1}^{\prime}} .
\end{aligned}
$$

If we take for example two modes with the maximum photon number, $N_{\max }=2$, we have the Fock state basis elements

$$
\left|n_{1}, n_{2}\right\rangle \in\{|0,0\rangle,|1,0\rangle,|0,1\rangle,|2,0\rangle,|1,1\rangle,|0,2\rangle\} .
$$

In this case the annihilation operators are of the form:

$\hat{a}_{2}=\left(\begin{array}{c|cc|ccc|cccc}0 & 1 & 0 & 0 & 0 & 0 & 0 & 0 & 0 & 0 \\ \hline 0 & 0 & 0 & \sqrt{2} & 0 & 0 & 0 & 0 & 0 & 0 \\ 0 & 0 & 0 & 0 & 1 & 0 & 0 & 0 & 0 & 0 \\ \hline 0 & 0 & 0 & 0 & 0 & 0 & \sqrt{3} & 0 & 0 & 0 \\ 0 & 0 & 0 & 0 & 0 & 0 & 0 & \sqrt{2} & 0 & 0 \\ 0 & 0 & 0 & 0 & 0 & 0 & 0 & 0 & 1 & 0 \\ \hline 0 & 0 & 0 & 0 & 0 & 0 & 0 & 0 & 0 & 0 \\ 0 & 0 & 0 & 0 & 0 & 0 & 0 & 0 & 0 & 0 \\ 0 & 0 & 0 & 0 & 0 & 0 & 0 & 0 & 0 & 0 \\ 0 & 0 & 0 & 0 & 0 & 0 & 0 & 0 & 0 & 0\end{array}\right)$,

$$
\hat{a}_{3}=\left(\begin{array}{c|cc|ccc|cccc}
0 & 0 & 1 & 0 & 0 & 0 & 0 & 0 & 0 & 0 \\
\hline 0 & 0 & 0 & 0 & 1 & 0 & 0 & 0 & 0 & 0 \\
0 & 0 & 0 & 0 & 0 & \sqrt{2} & 0 & 0 & 0 & 0 \\
\hline 0 & 0 & 0 & 0 & 0 & 0 & 0 & 1 & 0 & 0 \\
0 & 0 & 0 & 0 & 0 & 0 & 0 & 0 & \sqrt{2} & 0 \\
0 & 0 & 0 & 0 & 0 & 0 & 0 & 0 & 0 & \sqrt{3} \\
\hline 0 & 0 & 0 & 0 & 0 & 0 & 0 & 0 & 0 & 0 \\
0 & 0 & 0 & 0 & 0 & 0 & 0 & 0 & 0 & 0 \\
0 & 0 & 0 & 0 & 0 & 0 & 0 & 0 & 0 & 0 \\
0 & 0 & 0 & 0 & 0 & 0 & 0 & 0 & 0 & 0
\end{array}\right) .
$$

In calculating the effective Hamiltonian the operators are initially truncated at total photon number $N_{\max }+1$. This allows us to calculate an effective Hamiltonian and related scattering operator that accounts for states with $N=3$ photons that may contribute to states at $N=2$. For example the state $|3,0\rangle$ is not in the truncated basis described by Eq. B.3. however, $\hat{a}_{2}|3,0\rangle=\sqrt{2}|2,0\rangle$, is an element of the basis. Furthermore, this extended space ensures that the finite creation and annihilation operators have the correct commutation relations, i.e $\left[\hat{a}_{i}, \hat{a}_{j}^{\dagger}\right]=\delta_{i j}$ With this extended Hamiltonian calculated we can then truncate to the appropriate dimensions of the basis with $N_{\max }=2$. We calculate all of these matrices and all of the numerical examples in text with Mathematica computer software 22 .

\section{Appendix C. Secondary Example}

In this section we consider a secondary numerical example numerical similar to that presented in section 5. In this example we consider the case where the background field has OAM $\ell_{b}=+1$. The field profile of which is illustrated in figure 1(b). In 


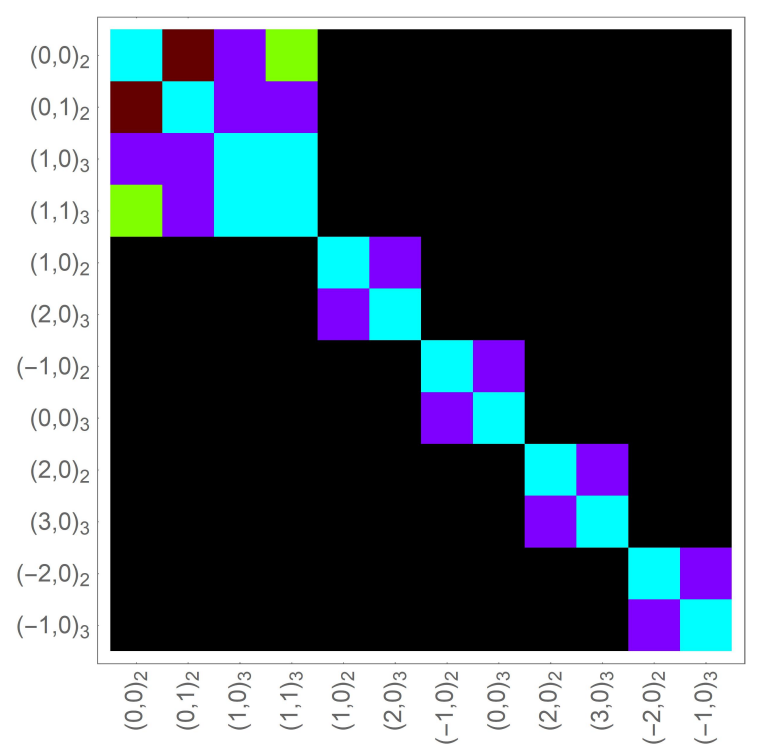

Figure C.4: Classical scattering matrix for $\ell_{b}=+1$ with indices $(\ell, p)_{\tau}$, represented on the false colour map shown in figure 1 (f)

this case the basis set is truncated at $2 p+\ell=2$ and is not closed with respect to the selection rule, $\ell_{3}=\ell_{2}+\ell_{b}$. Indeed, if we consider the case of the field with $\ell_{2}=2$ interacting with the background which has $\ell_{b}=+1$; the resulting field would have OAM $\ell_{3}=+3$, which is out with our truncated basis. In order to keep all possible interactions closed with respect to the basis we shift the $f_{3}(x, y, z)$ basis elements by $\ell=+1$.

The scattering matrix for this example is shown in figure C.4. As with the example presented in section 5, the matrix is block diagonal due to the conservation of OAM, i.e $\ell_{3}-\ell_{2}=\ell_{b}$. Again, similar to the example of section 5 the scattering matrix contains all of the symmetry associated with the system and is identical to the scattering operator, $\hat{S}$, if the system consists of a single photon, $N_{\max }=1$. This is highlighted in figure C.5.

It is clear that by changing the OAM of the background and shifting the basis fields appropriately, the eigenmodes of this matrix encode all of the symmetry in the scattering matrix. Consequently, diagonalising this scattering matrix will decouple the scattering operator as described in section 4 giving a diagonal unitary operator, $\hat{S}_{b}$, that acts on the Fock space as shown in figure C.5. This is true for any background field in any basis, we shift the basis elements here to present the most symmetric case. 

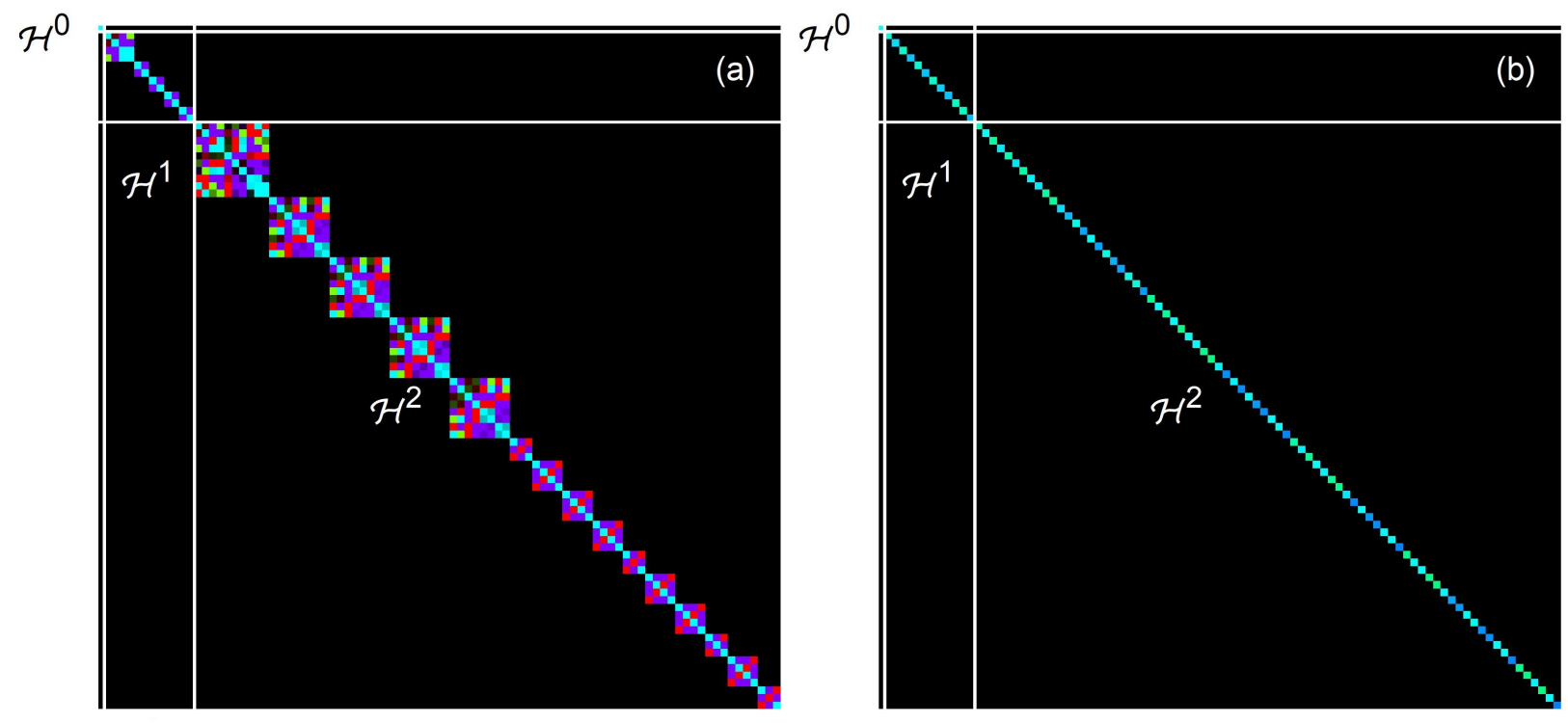

Figure C.5: Unitary scattering operator (a) $\hat{S}$ and (b) $\hat{S}_{b}$ represented with false colour space with false colour map shown in in figure 1 (f). The labels $\mathcal{H}^{n}$ correspond to the the $\mathrm{n}$ photon number Hilbert space. 